\title{
Spin trapping experiments with ethyl-substituted EMPO derivatives (EEMPO)
}

\author{
Klaus Stolze*1, Nataliya Rohr-Udilova ${ }^{2}$, Thomas Rosenau ${ }^{3}$, \\ Andreas Hofinger ${ }^{3}$ and Hans Nohl ${ }^{1}$
}

\author{
Address: ${ }^{1}$ Molecular Pharmacology and Toxicology Unit, Department of Natural Science, University of Veterinary Medicine, Vienna, Austria, \\ ${ }^{2}$ Division of Gastroenterology and Hepatology, Clinic of Internal Medicine III, Medical University of Vienna, Austria and ${ }^{3}$ Department of \\ Chemistry, University of Natural Resources and Applied Life Sciences (BOKU), Vienna, Austria \\ Email: Klaus Stolze* - klaus.stolze@vu-wien.ac.at \\ * Corresponding author
}

\author{
from 13th Scientific Symposium of the Austrian Pharmacological Society (APHAR). Joint Meeting with the Austrian Society of Toxicology (ASTOX) and the \\ Hungarian Society for Experimental and Clinical Pharmacology (MFT) \\ Vienna, Austria. 22-24 November 2007 \\ Published: 14 November 2007 \\ BMC Pharmacology 2007, 7(Suppl 2):A52 doi:10.1 I86/I47/-2210-7-S2-A52
}

This abstract is available from: http://www.biomedcentral.com/I47I-22/0/7/S2/A52

(C) 2007 Stolze et al; licensee BioMed Central Ltd.

Free radicals in biological systems play a major role in the onset of many diseases, e.g. oxygen-centered radicals such as hydroxyl or superoxide radicals. In order to identify and localize these radicals a series of four novel spin traps have been developed and their structure fully characterized by $\left[{ }^{1} \mathrm{H}\right]-$ and $\left[{ }^{13} \mathrm{C}\right]-N M R$ spectroscopy as well as mass spectrometry. The novel compounds can be described as ethylsubstituted EMPO derivatives, namely 5-ethoxycarbonyl3-ethyl-5-methyl-pyrroline $N$-oxide (3,5-EEMPO), 5ethoxycarbonyl-4-ethyl-5-methyl-pyrroline $\mathrm{N}$-oxide (4,5EEMPO), 5-ethoxycarbonyl-5-ethyl-3-methyl-pyrroline $\mathrm{N}$-oxide (5,3-EEMPO) and 5-ethoxycarbonyl-5-ethyl-4methyl-pyrroline $\mathrm{N}$-oxide (5,4-EEMPO). Their spin trapping behaviour towards a series of different oxygen- and carbon-centered radicals is described. All compounds were obtained in two different stereochemical forms (cis and trans), but only 3,5-EEMPO and 5,3-EEMPO could be separated into the different diastereomers using conventional chromatographic procedures. The cis- and transforms exhibited considerably different spectral parameters and stabilities of the respective superoxide adducts (ranging from about 12 to $35 \mathrm{~min}$ ). In addition, spin adducts obtained from different carbon-centered radicals derived from methanol, ethanol, formic acid and linoleic acid hydroperoxide have also been characterized. 